\title{
OBTENCIÓN DE DETERGENTE LÍQUIDO USANDO SAPONINA DE QUINUA (Chenopodium quinoa Willd), CHOCHO (Lupinus mutabilis Sweet) CABUYA (Sisalana perrine) Y SU DISEÑO DE PRODUCCIÓN
}

\author{
Obtaining liquid detergent using quinoa saponine (chenopodium quinoa willd), chocho \\ (lupinus mutabilis sweet) cabuya (sisalana perrine) and its production design
}

\author{
Mario-Gustavo Villacrés Alvarez, Cristina-Gabriela Calderón Tapia*, Lourdes-María Cauja \\ Moyón, Talía-Manuela Arcos Guamán.
}

Facultad de Ciencias Escuela Superior Politécnica de Chimborazo ESPOCH, Riobamba, Ecuador.

*cristy.gct@gmail.com

\begin{abstract}
Desde la aparición de la industria, así como obtenemos productos de mejor uso, también experimentamos cambios que perjudican nuestros recursos hídricos. Un ejemplo muy claro de ello es la presencia de tensoactivos en los detergentes, que por su naturaleza forman una capa de grasa en la superficie evitando el paso del oxígeno hacia el interior, provocando la muerte de las especies acuáticas, y sus restos al descomponerse emanan olores fuertes.

La industrialización de la quinua se realiza por vía seca que se conoce como escarificación, el polvillo es desechado sin uso alguno. Para el caso del chocho al realizar el lavado se elimina la saponina en su totalidad, en ríos, acequias o alcantarillado. Y en la industrialización de la cabuya en la producción de cuerdas, el zumo con alto contenido de saponina, también es desechado sin ninguna utilidad.

Por esta razón se diseñó un proceso para obtener un detergente líquido amigable con el medio ambiente, utilizando como materia prima los "desechos" generados en la industrialización de quinua, chocho y cabuya, utilizando su contenido de saponina que es biodegradable. La saponina se obtuvo por extracción simple utilizando como solvente etanol al $96 \%$ de pureza. Donde sus propiedades físico-químicas fueron: densidad 1,11g/mL; temperatura 20,2 ${ }^{\circ} \mathrm{C}$; $\mathrm{pH} 6,64$; IR 1,372; ${ }^{\circ}$ Brix 25 y viscosidad 386,8 cP.

El detergente obtenido se rigió bajo la Norma NTE INEN 0847 (2009), obteniendo de materia grasa un $3,28 \%$ de alcalinidad libre $(\mathrm{NaOH}) 0 \%$, de materia activa $36,12 \%$, fosfatos $0,08 \%$ y biodegradabilidad $94 \%$, lo que indica que es un producto libre de álcalis, silicatos de sodio anticorrosivos, estabilizadores de espuma de amida, carboxi metil celulosa para la formación de suspensiones de partículas de suelo, blanqueadores, suavizantes de tejidos, enzimas, abrillantadores ópticos y sulfato de sodio que son contaminantes, por lo tanto aportaría a la conservación ecológica del planeta, por lo que se puede comercializar, además se realizó los cálculos de los índices financieros dando como resultado un VAN de \$174.074,21 un TIR de $87 \%$ con un índice de rentabilidad de 2,82 siendo una muy buena opción para una microempresa por lo que se considera un proyecto totalmente viable
\end{abstract}

Palabras clave: Chenopodium quinoa Will, Lupinus mutabilis Sweet, Sisalana perrine, saponina, detergente.

Since the appearance of the industry, as well as we obtain products of better use, we also experience changes that damage our water resources. A very clear example of this is the presence of surfactants in detergents, which by their nature form a layer of fat on the surface, preventing the passage of oxygen into the interior, causing the death of aquatic species, and their remains when decomposing emanate strong smells.

The industrialization of quinoa is carried out by dry way that is known as scarification, the dust is discarded without any use. In the case of the lupine, when washing, the saponin is completely 
eliminated in rivers, ditches or sewers. And in the industrialization of the cabuya in the production of ropes, the juice with high content of saponin, is also discarded without any use.

For this reason a process was designed to obtain a liquid detergent friendly to the environment, using as raw material the "waste" generated in the industrialization of quinoa, lupine and cabuya, using its content of saponin that is biodegradable. Saponin was obtained by simple extraction using $96 \%$ ethanol as a solvent. Where its physical-chemical properties were: $1.11 \mathrm{~g} / \mathrm{mL}$ density; temperature $20.2^{\circ} \mathrm{C}$; $\mathrm{pH} 6.64$; IR 1,372 ; $^{\circ}$ Brix 25 and viscosity $386.8 \mathrm{cP}$.

The detergent obtained was regulated under the NTE INEN 0847 (2009) standard, obtaining $3.28 \%$ of free alkalinity $(\mathrm{NaOH}) 0 \%$, of active material $36.12 \%$, phosphates $0.08 \%$ and biodegradability $94 \%$. , which indicates that it is a product free of alkalis, anti-corrosive sodium silicates, amide foam stabilizers, carboxy methyl cellulose for the formation of suspensions of soil particles, bleaches, fabric softeners, enzymes, optical brighteners and sodium sulphate which are contaminants, therefore contribute to the ecological conservation of the planet, so it can be commercialized, in addition the calculations of the financial indexes were carried out, resulting in a NPV of $\$ 174,074.21$, an IRR of $87 \%$ with a profitability index of 2.82 , which is a very good option for a microenterprise. It is considered a totally viable project.

Key words: quinoa (Chenopodium quinoa Willd), chocho (Lupinus mutabilis Sweet), agave (Sisalana perrine), extraction, saponin, liquid detergent.

Fecha de recepción: 16-05-2018

Fecha de aceptación: 30-01-2019

\section{INTRODUCCIÓN}

La quinua (Chenopodium quinoa Willd) es una planta propia de la región andina, su cultivo puede soportar condiciones adversas como heladas, viento, sequía etc., (1).

Desde hace cientos de años se la utiliza como alimento, y existen varios estudios que muestran sus propiedades beneficiosas como por ejemplo antimicrobianas y antioxidantes (2).

Pero existen otras utilidades que se están estudiando, como la extracción saponina a partir de sus tallos, utilizando el proceso a presión de agua caliente (PHWE) obteniendo resultados alentadores (3), sin embargo no se ha intentado extraer la saponina con los residuos del proceso de escarificación.

El chocho Lupinus mutabilis Sweet también es un referente alimenticio de las comunidades indígenas del Ecuador, existen escasas publicaciones para el uso del mismo que no sea como antimicrobiano o alimento, hay cierto estudio donde se le usa para biochar, (4), pero no hay registros del uso de las saponinas provenientes del chocho.

La tercera especie en cuestión es el agave (Sisalana perrine), una planta con fibras duras, que representan el 3-5\% de su peso y se utilizan para elaboración de sogas, el 95$97 \%$ restante se conoce como desecho de sisal, que hace años no tenía uso, pero se descubrió que contiene saponinas esteroidales que pueden usarse potencialmente en formulaciones de alimentos, cosméticos y productos farmacéuticos, así como para la biorremediación del suelo (5).

Por otro lado la problemática de los detergentes es su formulación, la cual incluye intercambiadores de iones, álcalis (carbonato de sodio), silicatos de sodio anticorrosivos, estabilizadores de espuma de amida, carboxi metil celulosa para la formación de suspensiones de partículas de suelo, blanqueadores, suavizantes de tejidos, enzimas, abrillantadoras ópticas, fragancias y sulfato de sodio como diluyente (6).

Ciertos detergentes poseen en su composición cadenas alquílicas altamente ramificadas, extremadamente resistentes al ataque microbiano, por lo que son poco biodegradables, por éste efecto al entrar en contacto con medios acuáticos naturales, dan lugar a la formación de espumas en ríos y lagos, provocando pérdidas en la fauna y flora del lugar. (7) 
Dichos antecedentes han despertado el interés en varios investigadores para crear productos biodegradables, que no generen un gran impacto en el ecosistema.

Si se sustituye el dodecillbencensulfonato de sodio de los detergentes comunes por moléculas orgánicas esteroides o triterpenos unidos a un azúcar hidrófilo, se obtendría un detergente biodegradable (8). La saponina es una molécula con glucósidos triterpénicos y se encuentra presente en el chocho, quinua y cabuya. Por lo que en el presente estudio se pretende establecer protocolos de extracción de saponina a nivel industrial y diseñar un proceso para la obtención de un detergente biodegradable, que contribuya a la disminución de la contaminación producida por los detergentes. (9)

\section{MATERIAL Y MÉTODOS}

Se utilizó el desecho obtenido de la escarificación de la quinua de la industria Maquita ubicada en la región Sierra del Ecuador, el chocho se trasladó desde la parroquia Tixán y la cabuya se obtuvo de plantaciones ubicadas en San Gerardo del cantón Guano. Se realizó de forma manual la selección de la muestra y limpieza de la materia prima utilizando la norma NTE INEN 1233 (1995) (10).

Para la extracción de saponina se utilizó el método de destilación simple, en la cabuya un pre tratamiento de la muestra, para lo cual se realizó un proceso de extrusión utilizando un molino. Luego el zumo resultante de las muestras de chocho, cabuya y quinua se dejaron remojar durante dos días en etanol al 96\% de pureza.

\section{Filtración}

La etapa de filtración garantiza que no haya partículas sólidas que puedan alte- rar las propiedades del detergente. En el zumo de cabuya se produce una torta con un espesor considerable por lo cual se utiliza un filtro prensa y se obtiene un zumo clarificado, es muy importante tener en cuenta esta fase, para que posteriormente en la etapa de filtración no se queme la muestra. (11)

\section{Destilación del zumo de cabuya quinua y chocho para obtener saponina}

En la etapa de destilación se tiene en cuenta la temperatura a la que debe ebullir el zumo (12), dado que si sobrepasa los $100^{\circ} \mathrm{C}$ existe un desequilibrio de la saponina, produciéndose burbujas, generando el aumento de presión, provocando la subida de la saponina hacia el condensador, y posterior pérdida de la materia prima para la elaboración del detergente. En esta fase se puede recuperar el solvente, para reducir costos de producción. (13)

\section{Segunda Filtración}

La segunda filtración se dará dependiendo de la composición de la cabuya, por si se forme al final del destilado una capa de grasa que debe filtrarse para evitar grumos en el detergente. (14)

\section{Análisis de saponina}

Para determinar si la saponina está en óptimas condiciones se procede a su caracterización físico química, tabla 1. (10)

\begin{tabular}{|l|r|}
\hline PARÁMETRO & RESULTADO \\
\hline Densidad & $1,11 \mathrm{~g} / \mathrm{mL}$ \\
\hline Temperatura & $20,2^{\circ} \mathrm{C}$ \\
\hline pH & 6,64 \\
\hline Índice de refracción & 1,372 \\
\hline Grados Brix & 25 \\
\hline Viscosidad & $386,8 \mathrm{cP}$ \\
\hline Método de espuma (tubo de $\mathbf{1 5} \mathbf{~ c m}$ ) & $8 \mathrm{~cm}$ \\
\hline
\end{tabular}

Tabla 1. Resultados de los análisis físico -químicos de la saponina

\section{Mezclado}

Se añaden aditivos al detergente tales como: Texapón, vinagre de manzana, colorante natural y aroma (15), agitando de manera vigorosa por 20 minutos en cada aditivo utilizado para que la homogenización sea total. (16) 


\section{Análisis del detergente}

Se realizó análisis fisicoquímicos del detergente líquido obtenidos como son: materia activa, materia grasa, alcalinidad total, fosfatos, $\mathrm{pH}$, Biodegradabilidad, tabla 2 .

\begin{tabular}{|l|r|}
\hline PARÁMETRO & \multicolumn{2}{|l|}{ RESULTADO } \\
\hline Densidad & $1,07-1,11 \mathrm{~g} / \mathrm{mL}$ \\
\hline Temperatura & $20^{\circ} \mathrm{C}$ \\
\hline pH & $7,0-9,0$ \\
\hline Viscosidad & $10000 \mathrm{cP}$ \\
\hline
\end{tabular}

Tabla 2 Resultados de los análisis físico-químicos del Texapón N70 A.

\section{Envasado y almacenamiento}

$\mathrm{Al}$ realizar un análisis de estrategia de marketing se utilizó envases de 1 litro y 1 galón, siendo las presentaciones más comunes que están en el mercado.

Una vez envasado, según el diseño de la planta de producción propuesto ver Figura 1, el producto irá al área de almacenamiento y posteriormente se realizará la distribución a los distintos puntos de venta. (17)

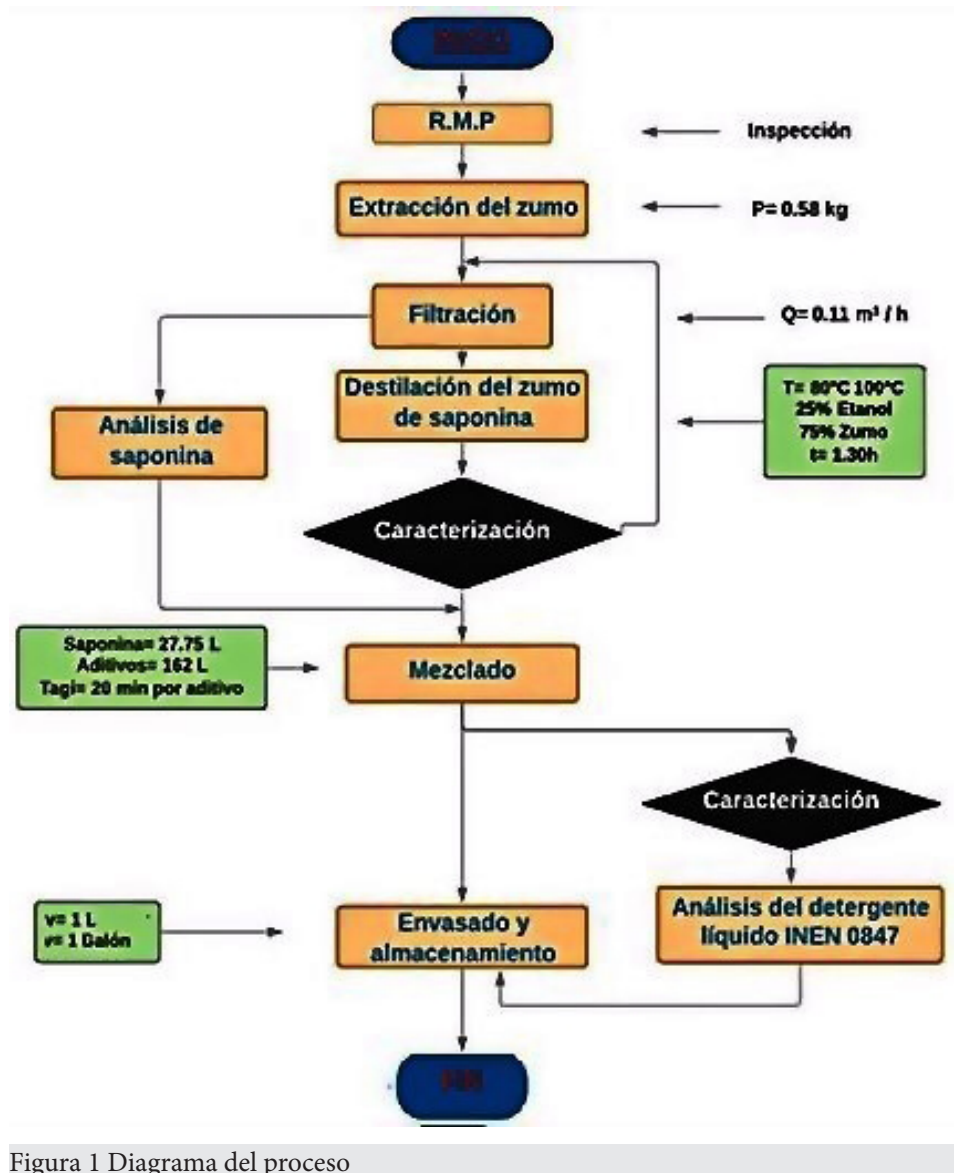

\section{RESULTADOS Y DISCUSIÓN}

El método de destilación simple utilizando como solvente etanol al $96 \%$ de pureza, es el método convencional (3), que brinda ventajas frente a otros métodos de extracción como soxlheth, liofilización, evaporación en baño maría o estufa.

Con el método de destilación simple se obtuvo un tiempo de extracción de 1.30 horas, el solvente utilizado es recuperable, lo cual es importante para una producción a gran escala. Las muestras no se contaminan, lo que si sucede con el método soxlheth. Y lo más importante, se obtiene una saponina en estado líquido que facilita la mezcla en la preparación del detergente líquido, y con los otros métodos se obtiene una saponina en estado sólido.

En la tabla 1 se muestran los resultados de los análisis físico-químicos de la saponina, sin embargo la saponina en la actualidad no tiene una normativa vigente ecuatoriana, entonces se puede comparar con los valores del Texapón N70A que se muestran en la tabla 2.

Como se observa estos parámetros están dentro de rango permitido, y al momento de mezclar la saponina con el texapón se obtuvo una mezcla totalmente homogénea.En la caracterización físico-química final del detergente líquido que se observa en la tabla 3 , conforme a la norma NTE INEN 0847 se obtuvo: 36,12\% de materia activa que es claramente la saponina, $94 \%$ de biodegradabilidad ya que se utilizó la saponina sin añadir compuestos inorgánicos, garantizando un detergente libre de: álcalis y fosfatos que se puede desechar en las vertientes sin causar un efecto nocivo al medio ambiente (19).

Los resultados se compararon con tres tipos de detergentes industriales como se muestra en la tabla 4 , que fueron uti- 
lizados en derrames de crudos livianos, sin embargo se puede observar que la biodegradabilidad de aquellos detergentes son menores que el obtenido.

\begin{tabular}{|c|c|}
\hline PARÁMETRO & RESULTADO \\
\hline Materia grasa & $3,28 \%$ \\
\hline Alcalinidad Libre & $0 \%$ \\
\hline pH & 1,34 \\
\hline Materia Activa & $36,12 \%$ \\
\hline Fosfatos & $0,08 \%$ \\
\hline Biodegradabilidad & $94 \%$. \\
\hline
\end{tabular}

Tabla 3. Resultados de los análisis del detergente líquido

\begin{tabular}{|l|c|}
\hline DETERGENTE & BIODEGRADABILIDAD \\
\hline D.IndustrialN4 - 3G & $80 \%$ \\
\hline D.IndustrialN4 - 3M & $80 \%$ \\
\hline D.IndustrialN4 - 3Ce2 & $75 \%$ \\
\hline
\end{tabular}

industrial

\section{Dimensionamiento de los equipos a escala indus- trial para la obtención del detergente}

Los equipos necesarios para la obtención de detergente líquido se muestra en la Figura 2. La cual está dada para una producción de 189,25L/h de detergente líquido (20)

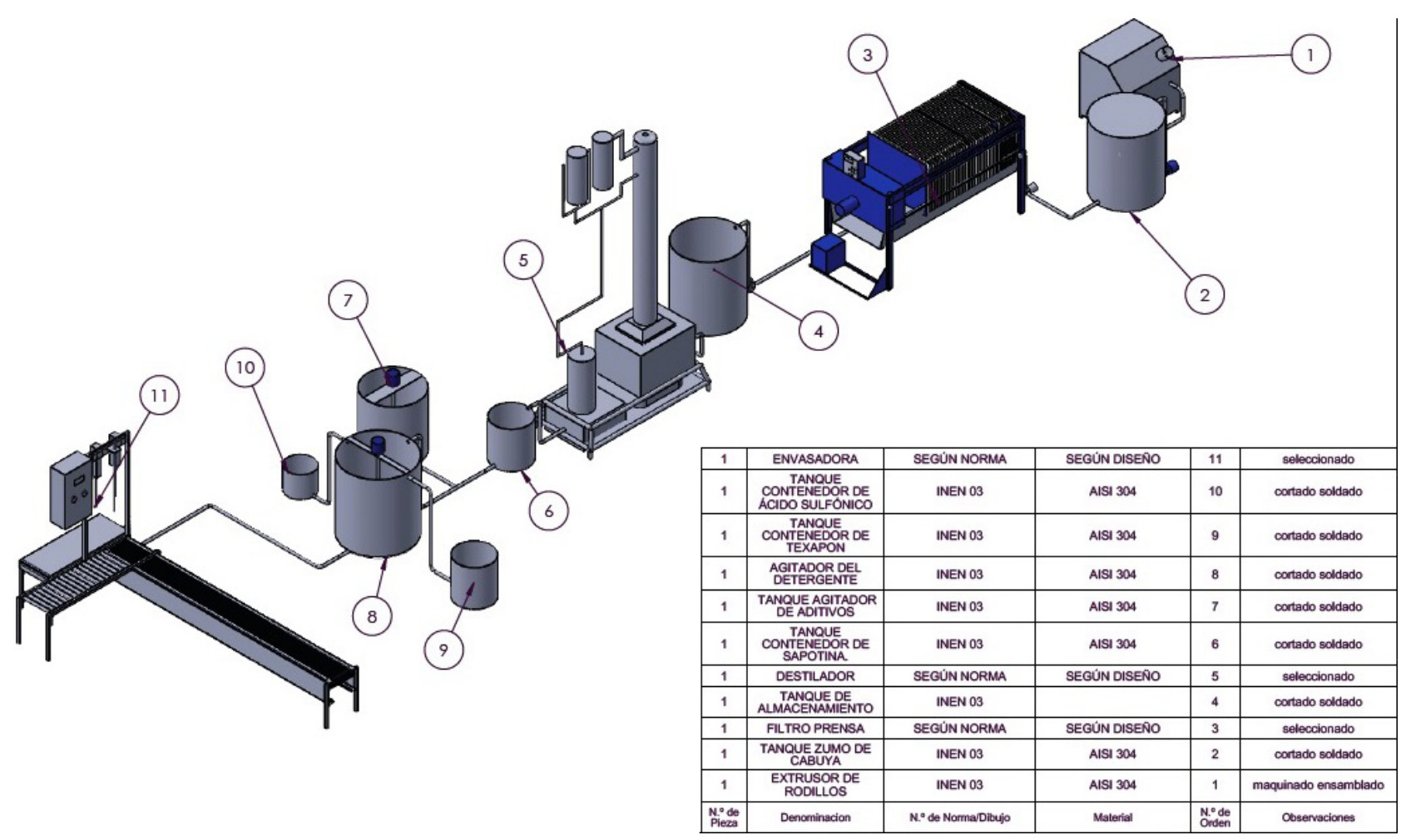

Figura 2 Diagrama de los equipos utilizados en el proceso.

Estimación del presupuesto y viabilidad del proyecto

Se realizó un análisis financiero ilustrado en la tabla 5, para verificar si este proyecto es viable o no, se hace uso del cálculo de los indicadores financieros, VAN (Valor Actual Neto) \$174.074,21.

La Tasa Interna de Retorno al implementar este proyecto sería del $87 \%$. I. Renta- bilidad \$2,82 esto nos indica que por cada dólar invertido se obtendrá una ganancia neta de 1,82 ctvs., siendo un valor muy considerable de ganancia y el periodo de recuperación de lo invertido será de 1 año con dos meses.

Por esta razón se considera que este proyecto financieramente es viable, debido a la importancia ancestral de las plantas utilizadas como materia prima (22), el hecho de utilizar desechos de las mismas que darían un valor agregado al proceso, y finalmente por el producto obtenido con un alto índice de biodegradabilidad. 


\begin{tabular}{|c|c|c|c|c|c|c|c|c|c|}
\hline \multicolumn{4}{|c|}{ INVERSION INICIAL 77751,5 } & \multirow[b]{2}{*}{$\begin{array}{l}\text { Año de } \\
\text { Operación }\end{array}$} & \multirow[b]{2}{*}{$\begin{array}{l}\text { Ingresos } \\
\text { Totales }\end{array}$} & \multirow[b]{2}{*}{$\begin{array}{l}\text { Egresos } \\
\text { Totales }\end{array}$} & \multirow[b]{2}{*}{$\begin{array}{l}\text { Flujo } \\
\text { Efectivo } \\
\text { Neto }\end{array}$} & \multirow[b]{2}{*}{$\begin{array}{l}\text { Tasa de } \\
\text { Descuento } \\
10 \%\end{array}$} & \multirow[b]{2}{*}{ Acumulado } \\
\hline $\begin{array}{c}\text { ¿ Costo por } \\
\text { quipos }\end{array}$ & $\begin{array}{l}\text { Recursos } \\
\text { humanos }\end{array}$ & $\begin{array}{l}\text { Estructura } \\
\text { física }\end{array}$ & $\begin{array}{c}\text { Servicios } \\
\text { básicos }\end{array}$ & & & & & & \\
\hline \multirow[t]{9}{*}{26310} & 7687,500 & 93569,120 & 2800 & 1 & 157135,032 & 47050 & 110085,032 & 99076,529 & $-10280,621$ \\
\hline & & & & 2 & 162075,885 & 48436,580 & 113639,305 & 102275,375 & 91994,754 \\
\hline & & & & 3 & 169779,980 & 50738,958 & 119041,022 & 107136,919 & 146586,659 \\
\hline & & & & TOTAL & 488990,897 & 146225,538 & 342765,359 & 308488,823 & 228300,792 \\
\hline & & & & \multicolumn{6}{|c|}{ INDICADORES FINANCIEROS } \\
\hline & & & & \multicolumn{2}{|l|}{$\overline{\text { VAN }}$} & $\$ 174.074,21$ & \multicolumn{2}{|c|}{ VAN $>0$ Rentable } & Se Acepta \\
\hline & & & & \multicolumn{2}{|l|}{ TIR } & $87 \%$ & & & Se Acepta \\
\hline & & & & \multicolumn{2}{|c|}{$\begin{array}{ll}\text { INDICE } & \text { DE } \\
\text { RENTABILIDAD } & \end{array}$} & 2,821 & & & Se Acepta \\
\hline & & & & \multicolumn{2}{|c|}{$\begin{array}{l}\text { PERIODO } \\
\text { RECUPERACION }\end{array}$} & 1,2 años & & & \\
\hline
\end{tabular}

Tábla 5. Resultados del presupuesto del proyecto

\section{CONCLUSIONES}

El estudio macromorfológico de hojas, flores, frutos y semillas, y el estudio micromorfológico de flores, hojas y polen permitieron identificar y diferenciar cada una de las especies del género Passiflora presentes en la provincia de Chimborazo.

Esta investigación representa la etapa inicial del estudio comparativo de la actividad psicoactiva de especies de Passiflora de Chimborazo, las cuales presentan un alto potencial ansiolítico.

En etapas posteriores es necesario realizar análisis de la composición química y estudios de actividad farmacológica y toxicidad. 


\section{R}

1. Jacobsen, S. Mujica, A. \& Jensen, C. The Resistance of Quinoa (Chenopodium quinoa Willd.) to Adverse Abiotic Factors, Food Reviews International. 2003; 19(2): 99-109.

2. Vega, A. Zura, L. Lute, M. Jagus, R. Agüero, V. Pastén, A. Scala, K. \& Uribe, E. Assessment of Dietary Fiber, Isoflavones and Phenolic Compounds with Antioxidant and Antimicrobial Properties of Quinoa (Chenopodium quinoa Willd.); Chilean journal of agricultural \& animal sciences. 2018; 34 (1): 57-67.

3. Gil, A. Salas, D. Grey, C. Nordberg, E. Rodriguez, I. Linares, J. Integrated process for sequential extraction of saponins, xylan and cellulose from quinoa stalks (Chenopodium quinoa Willd.). Elsiever Industrial Crops and Products. 2018; 121(1): 54-65.

4. Heredia, M. Tarelhoa, L. Matosa, A. Robaina. M, Narváez, R. Peralta, M. Thermoeconomic analysis of integrated production of biochar and process heat from quinoa and lupin residual biomass. Elsevier Energy Policy. 2018; 114(1): 332-341.

5. Dias, B. Sales, D. Weingart, D. Zarur, M. Functional properties of saponins from sisal (Agave sisalana) and juá (Ziziphus joazeiro): Critical micellar concentration, antioxidant and antimicrobial activities. Elsevier Colloids and Surfaces A: Physicochemical and Engineering Aspects.2013; 436(5):736743.

6. Bailey, P. \& Bailey, C. Química Orgánica, Conceptos y aplicaciones. 5ta ed. México: Prentice Hall Hispanoamérica, S.A; 1998. p. 487-489.

7. Domínguez, M. La contaminación ambiental, un tema con compromiso social. Scielo P+L. 2015; 10(1): Caldas.

8. Gender, K. \& A, Juan. Estudio de la biodegradación de los detergentes comerciales domésticos de nuestro país. [Trabajo de titulacion de Pre grado]. Universidad de Guayaquil. Facultad de Ingeniería Química. Ingeniería Química; 2005.

9. Gozáles, M., Barrenetexea, C., Pérez, J., Rodriguez, B. Contaminación ambiental: una visión desde la química. España : Paraninfo; 2003.

10. ECUADOR. INSTITUTO NACIONAL ECUATORIANO DE NORMALIZACIÓN. Procedimiento para la Toma de Muestras de Granos y Cereales; 1995.

11. Stephen, J. \& Weiniger, F. Química Orgánica . Bacelona-España; 1988. p. 706.

12. Wade L, Jr. Química Orgánica. Madrid: Pearson Education, S.A; 2004. p. 1075-1077.

13. Guillot, D. \& Der, P. Agave sisalana Perr. Ex Engelm., y sus cultivares en España. Vol. 6. España: Bouteloua; 2009. p. 72-75.

14. Meyhuay, M. Quinua: Operaciones de Pos cosecha. AGSI/FAO [Internet]; 2013 [Citado: 15 septiembre 2017]. Disponible en: http://www.fao.org/docrep/018/ar364s/ar364s.pdf

15. Wilkinson, J. B. y Moore, R. J. Cosmetología de Harry. Madrid: Edigrafos, S. A; 1990. p. 941.

16. Geankopolis, C. J. Procesos de transporte y operaciones unitarias. México : Contiental, S.A ; 1998. p. 66 ; p. 161.

17. Warren L, McCabe, Smith, Julian C y Harrion, Peter. Operaciones Unitarias en Ingeniería Química. España : McGraw-Hill; 1991. p. 242 ; p. 890.

18. Lucid Software Inc. (2018). Lucidchart-flowchart maker. [Internet]; 2018. [Citado: 25 octubre 2017] Disponible en: https://www.lucidchart.com/.

19. ECUADOR. INSTITUTO NACIONAL ECUATORIANO DE NORMALIZACIÓN (1982). Requisitos que debe Cumplir el Detergente Líquido Destinado al Uso Doméstico Manual de Limpieza y puesta a punto de pisos y zonas comunes en alojamientos. Málaga : Vértice; 2009.

20. Ocon, J. \& Tojo, G.. Problemas de Ingeniería Química Operaciones Básicas Tomo I. Madrid: Aguilar; 1963. p. 280.

21. Dassault Systemes SolidWorks Corporation. SolidWorks-Herramientas. [Internet] 2018. [Citado: 25 octubre 2017] Disponible en: http://www.solidworks.es/sw/3d-cad-design-software.htm

22. Peralta, E., Mazón, N., Murillo, A., Rodríguez, D., Lomas, L., y Monar, C. Manual Agrícola de Granos Andinos Chocho, Quinua, Amaranto y Ataco. Vol. 1. 3a ed. Ecuador: INIAP; 2012. p. 2-8. 